

\title{
A framework for creating population specific multimodal brain atlas using clinical T1 and diffusion tensor images
}

Vikash Gupta, Grégoire Malandain, Nicholas Ayache, Xavier Pennec

\section{To cite this version:}

Vikash Gupta, Grégoire Malandain, Nicholas Ayache, Xavier Pennec. A framework for creating population specific multimodal brain atlas using clinical T1 and diffusion tensor images. MICCAI 2015 Workshop on Computational Diffusion MRI (CDMRI'15), Oct 2015, Munich, Germany. pp.99-108, 10.1007/978-3-319-28588-7_9. hal-01261115

\section{HAL Id: hal-01261115 \\ https://hal.inria.fr/hal-01261115}

Submitted on 23 Jan 2016

HAL is a multi-disciplinary open access archive for the deposit and dissemination of scientific research documents, whether they are published or not. The documents may come from teaching and research institutions in France or abroad, or from public or private research centers.
L'archive ouverte pluridisciplinaire HAL, est destinée au dépôt et à la diffusion de documents scientifiques de niveau recherche, publiés ou non, émanant des établissements d'enseignement et de recherche français ou étrangers, des laboratoires publics ou privés. 


\title{
A framework for creating population specific multimodal brain atlas using clinical $\mathrm{T} 1$ and diffusion tensor images
}

\author{
Vikash Gupta*, Gregoire Malandain ${ }^{\star \star}$, Nicholas Ayache, and Xavier Pennec \\ INRIA Sophia Antipolis - ASCLEPIOS Project, \\ 06902 Sophia Antipolis Cedex, France.
}

\begin{abstract}
Spatial normalization is one of the most important steps in population based statistical analysis of brain images. This involves normalizing all the brain images to a pre-defined template or a population specific template. With multiple emerging imaging modalities, it is quintessential to develop a method for building a joint template that is a statistical representation of the given population across different modalities. It is possible to create different population specific templates in different modalities using existing methods. However, they do not give an opportunity for voxelwise comparison of different modalities. A multimodal brain template with probabilistic region of interest (ROI) definitions will give opportunity for multivariate statistical frameworks for better understanding of brain diseases. In this paper, we propose a methodology for developing such a multimodal brain atlas using the anatomical T1 images and the diffusion tensor images (DTI), along with an automated workflow to probabilistically define the different white matter regions on the population specific multimodal template. The method will be useful to carry out ROI based statistics across different modalities even in the absence of expert segmentation. We show the effectiveness of such a template using voxelwise multivariate statistical analysis on population based group studies on HIV/AIDS patients.
\end{abstract}

Keywords: Multimodal brain atlas, Probabilistic white matter parcellation map, HIV, DTI-T1 brain template

\section{The need for a probabilistic multimodal atlas}

The growth in brain imaging data across different modalities gives an opportunity to understand the disease progression and make correlations across them. Statistical analysis across different modalities and across population require spatial normalization. All the brain images are often normalized to a pre-defined template, for example the ICBM-152 or MNI template. However in [1] and [2], the authors have shown that choosing a generic template biases the statistical

\footnotetext{
* presently at Imaging Genetics Center, University of Southern California

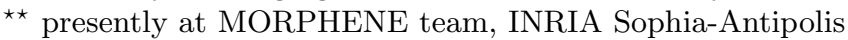


results. For example, if one intends to do statistical analysis on a population of children, choosing an MNI template will involve an unintended scaling of all the brain images in the population adding a bias to the results. Moreover the generic MNI-T1 template is not a statistical representation of the population under consideration. The importance of multimodal brain atlases is discussed in detail in [3]. We support the need of such a multimodal template by comparing two groups of population. One of the groups comprises 18 healthy controls without any episodes of neurological disorder and another group of 30 patients with HIV associated neurocognitive disorder.

Some of the most common atlases being used today for spatial normalization are the ICBM (International Consortium of Brain Mapping), the MNI (Montreal Neuroimaging Institute) atlas and the FMRIB58_FA atlases. The ICBM initiative has provided the neuroimaging community with a number of brain atlases. The three atlases which we will like to discuss here are the nonlinear version of ICBM T1 atlas, ICBM DTI-81 atlas and the white matter parcellation map (WMPM) [4]. It should be noted that though the atlases are in the MNI coordinate space, they are not necessarily built with the same population. Thus, the DTI atlas and the T1 atlas do not represent the variability across different modalities in the same population and so are not suited for a multimodal statistical analysis. There are two different versions of the ICBM T1 template. The first one was built in 2001 using an affine registration which was followed by another one built in 2009 using affine and nonlinear image registration. The 2009 version presents a more detailed outline of different brain structures as compared to its previous version ${ }^{1}$. Some of the drawbacks of the present ICBM atlases are as follows,

1. The DTI atlas was affinely aligned with the 2001 affine version of ICBM T1 template.

2. For creating the DTI atlas, scalar averaging of tensor elements was performed.

3. The DTI-81 data is normalized using an affine registration which increases the chances of misalignment of different brain structures.

4. The ICBM DTI-81 and ICBM 152 nonlinear atlases are independent atlases in their own right. However, the atlases cannot be used for a multimodal study because they are not aligned in the same geometrical space.

In the following sections, we present an automated workflow to build a multimodal brain atlas using DTI and T1 images. Unlike the ICBM atlases, this atlas is in the same coordinate space which allows voxelwise comparison across the two modalities. In addition to the multimodal template we also present a method to probabilistically transfer the white matter labels in our template space. This opens further room for ROI based statistics on white matter region. Out of many possible applications to the presented atlas, we illustrate the applicability and relevance of the method by building a multimodal template to compare groups of HIV/AIDS patients with controls.

\footnotetext{
${ }^{1}$ The ICBM family of templates are available for download at http://www. loni.usc. edu/atlases/Atlas_Detail.php?atlas_id=5
} 


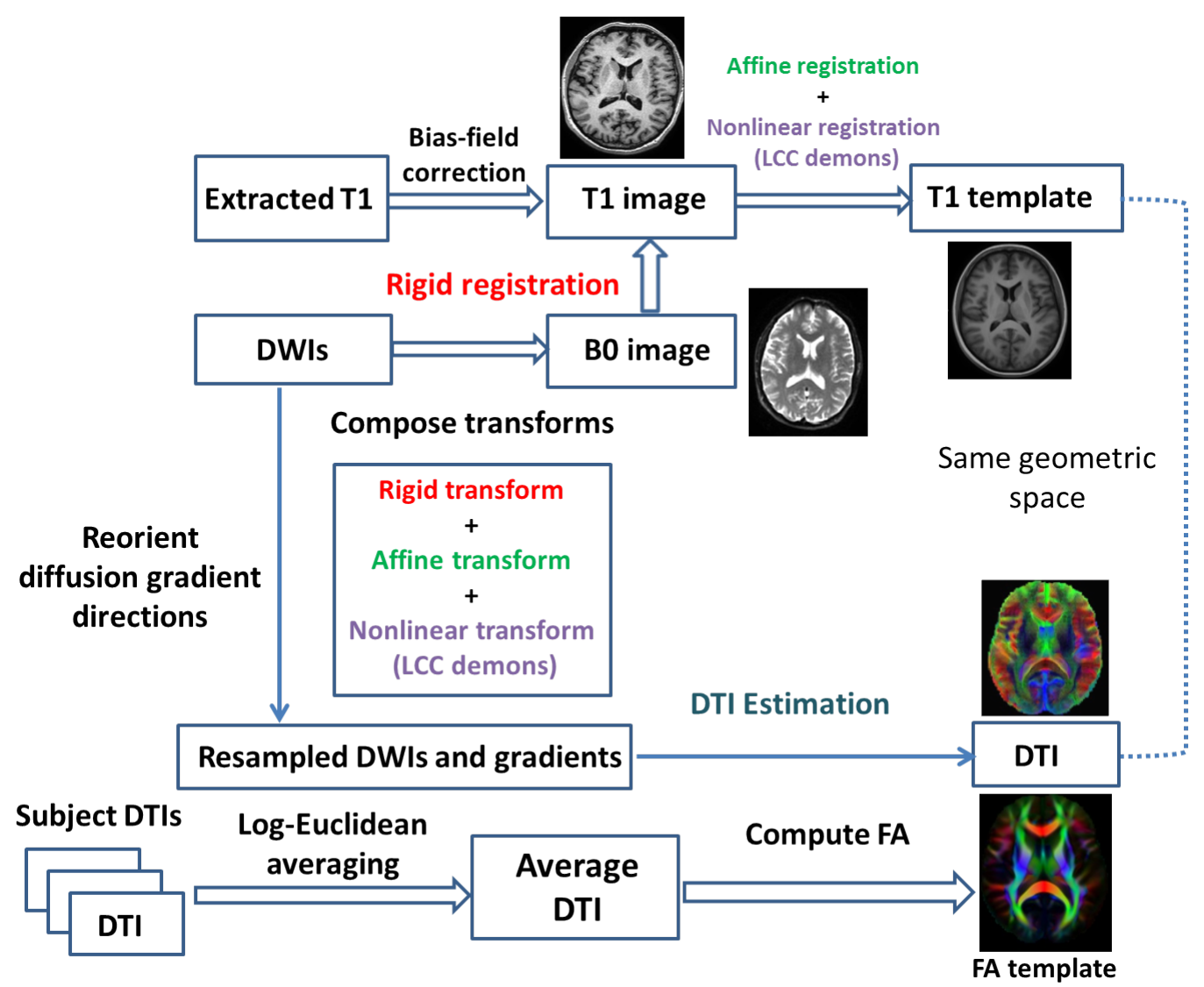

Fig. 1. Workflow for generating joint T1 and DTI brain atlas. Registrations and the corresponding transforms are color matched

\section{Atlas construction}

\section{$2.1 \quad$ Joint T1 and DTI template}

An unbiased T1 atlas is constructed using the methodology outlined in [5] and [6]. In principle, a similar method can be used to make a DTI template from a given population. However, in such a case the DTI template and the T1 template will be in different spaces and a comparative study across different modalities cannot be performed. For constructing a joint T1 and DTI template, we propose a workflow that takes into account physically plausible transformations that exists in the image space and across modalities. The workflow is shown in figure 1. The dicom images were extracted using the MRIcron software into a nifti image format. The diffusion weighted images (DWIs) are corrected for eddy currents distortions and head motion using the FSL toolbox. N4ITK bias correction tool 
[7] was used for intensity bias correction in the T1 images. The undiffused $\left(\mathrm{B}_{0}\right)$ images and the T1 images are rigidly registered using FSL's flirt tool using seven degrees of freedom and mutual information cost function to take into account the multimodality of the images. Seven degrees of freedom for the registration takes into account the rigid motion of the head (rotation and translation) along with the scaling of the voxels that is present due to differences in the field of view of the $\mathrm{B} 0$ and the $\mathrm{T} 1$ images during image acquisition. The $\mathrm{T} 1$ images were first aligned to the $\mathrm{T} 1$ template using an affine followed by a nonlinear registration using LCC-demons algorithm [8]. The rigid transform between the subject's B0 and T1 images, the affine and nonlinear deformation field are composed in order to produce the net deformation field. The diffusion images are then resampled into the T1 template image space using the composed deformation field. The combined deformation field can be expressed using a displacement field $(u)$. The local linear transformation an be described as

$$
T=I+\frac{d u}{d x},
$$

where $I$ is the identity matrix and $\frac{d u}{d x}$ Jacobian of the deformation field at the point $x$. The Jacobian matrix can be decomposed into a rotation component $R$ and a deformation component $P$ using the polar decomposition theorem. Using singular value decomposition (SVD), the rotation matrix $R$ ca be computed as

$$
R=U V^{T},
$$

where $F=U W V^{T}$ is the singular value decomposition of the matrix $F$. The diffusion gradient directions are then transformed to the new space using the rotational component of the transformation for each voxel as

$$
g_{v}=R g^{T},
$$

where $g$ is the diffusion gradient direction and $g_{v}$ is the transformed gradient direction. The diffusion tensors are then estimated in the tensors are then estimated in the template space using the algorithm presented in [9]. Once the diffusion tensors are estimated, they are averaged using the Log-Euclidean framework [10] to compute the DTI template. This workflow produces a combined T1 and DTI template in a common geometrical space.

\subsection{Probabilistic white matter}

For computing ROI based statistical analysis, it is important to have an accurate segmentation of the white matter regions. However, an accurate segmentation is a difficult problem because of the partial volume effects in the images which makes the segmentation task particularly difficult. In such a scenario it is desirable to have an automated probabilistic segmentation of the ROIs. A probabilistic segmentation allows us to attach an additional level of accuracy depending on how conservative one is in choosing the ROIs, thus mitigating the problems 
due to misregistration to certain extent. For generating a probabilistic parcellation of the white matter, we used the celebrated ICBM-WMPM as a prior. The WMPM is defined in the ICBM-DWI template space. he ICBM-DTI and the ICBM-152 affine template are aligned and share the same geometric space. Thus a registration using the ICBM-152 template will approximate a registration between the ICBM-DWI and the ICBM-152 nonlinear template. The workflow for transferring the labels is shown in figure 2. The different steps involved in the transferring the labels is enumerated as,

1. The ICBM-152 affine template is affinely aligned with the 2009 ICBM-152 nonlinear template.

2. The ICBM-152 nonlinear template (moving image) is registered with the T1 image (fixed image) of each of the subject using an affine and nonlinear registration. The target image for the registration is the $\mathrm{T} 1$ image

3. Similarly, the subject T1 image (moving image) is registered with the populationspecific template (fixed image) created before.

4. All the transformations are composed in the same order for each subject.

5. The respective transformations for each subject are applied to the the ICBMWMPM for transferring the labels.

6. All the transferred labels are averaged to produce the probabilistic parcellation map.

All the anatomical T1 registrations are carried out using LCC-demons algorithm in both the workflows.

\section{Results}

A total of 18 controls and 30 patients with HIV associated neurocognitive disorder (HAND) were chosen for the study. The images were acquired using a 1.5 T MRI scanner (GE Signa HDxt ${ }^{\circledR}$ ). T1-weighted images were acquired using a magnetization prepared 3D Spoiled Gradient Recalled (SPGR) sequence (TR = $12.4 \mathrm{~ms}, \mathrm{TE}=5.2 \mathrm{~ms}, \mathrm{TI}=300 \mathrm{~ms}$, flip angle $=18^{\circ}, \mathrm{FOV}=240 \mathrm{~mm}$ ). The T1 images have $256 \times 256 \times 248$ voxels, with an isotropic voxel size of $0.6 \mathrm{~mm}$. The DTI data was acquired with 23 encoding gradient direction and one undiffused B0 image . The diffusion weighted images has $256 \times 256 \times 26$ voxels with sizes $0.9375 \times 0.9375 \times 5.5 \mathrm{~mm}^{3}$. The b-value for the acquisition was $700 \mathrm{~s} / \mathrm{mm}^{2}$.

\subsection{Multimodal template and probabilistic ROIs}

Figure 3.1 shows the multimodal template. The different structures across the two modalities T1 and DTI are in good agreement as shown in the figure. The top row shows the FA template (in red-yellow) overlayed on the anatomical T1 image. In the bottom row we show good agreement between the DTI and T1 templates. A close-up shows the diffusion tensors in genu and spelnium of the corpus callosum in detail. In order to facilitate ROI based statisitcs, all the 52 white matter labels defined in ICBM-WMPM are transferred to this population 


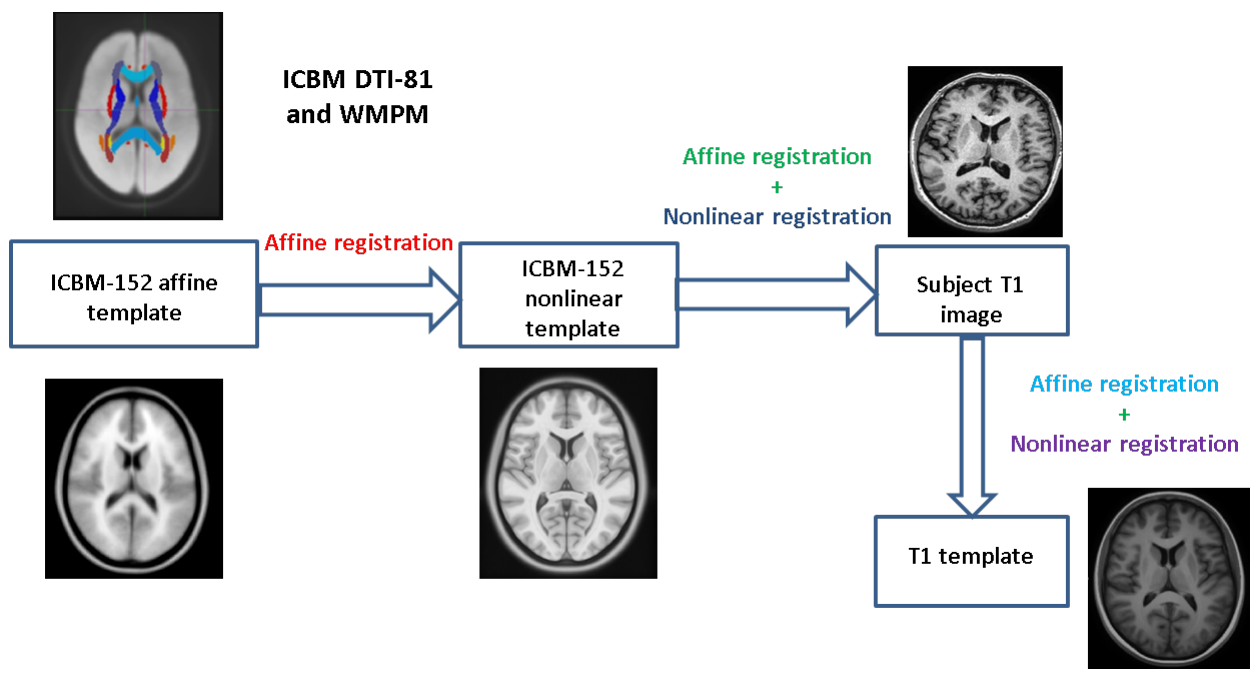

Fig. 2. Workflow for transferring white matter labels in ICBM-WMPM to a population specific multimodal atlas. The ICBM DTI-81 and the ICBM 152-affine atlas are defined in the same geometry. The ICBM-152 affine template is affinely registered with the ICBM-152 nonlinear template. The ICBM nonlinear template is then registered with each of the subject's T1 image which is again registered with the population specific T1 templated The arrows show the registration paths and the corresponding registration methods are shown above the arrows.

specific template. In figure 4 probabilistic ROIs for external and internal capsule, middle cerebellar peduncle (MCP) and corpus callosum is shown. It also shows a probabilistic iso-surface rendition of the MCP and corpus callosum. The probability of a voxel being classified in a certain ROI is highest in the center and decreases outwards as expected from blue to red. Such renditions can be used for shape analysis of individual white matter structures.

\subsection{Statistical analyses on HIV patients}

A multimodal population specific brain atlas can be used for multivariate statistical analysis. As opposed to the univariate analysis, in this case it is possible to combine information from different modalities which increases the statistical power of the test. In univariate analysis like tract based spatial statistics (TBSS) or voxel based morphometry, the focus of study is to find changes in a single tissue type. In order to illustrate one possible application of such a multimodal template, we use the FA images from the DTIs and log-Jacobian from the T1 registration. FA images contain information about the white matter integrity, where as the logarithm of the Jacobian determinant of the deformation field gives information about local volume shrinkage or expansion. For conducting the statistical test, first a multimodal template is created as mentioned above. All DTIs and T1 images are registered to the common template space. FA maps 

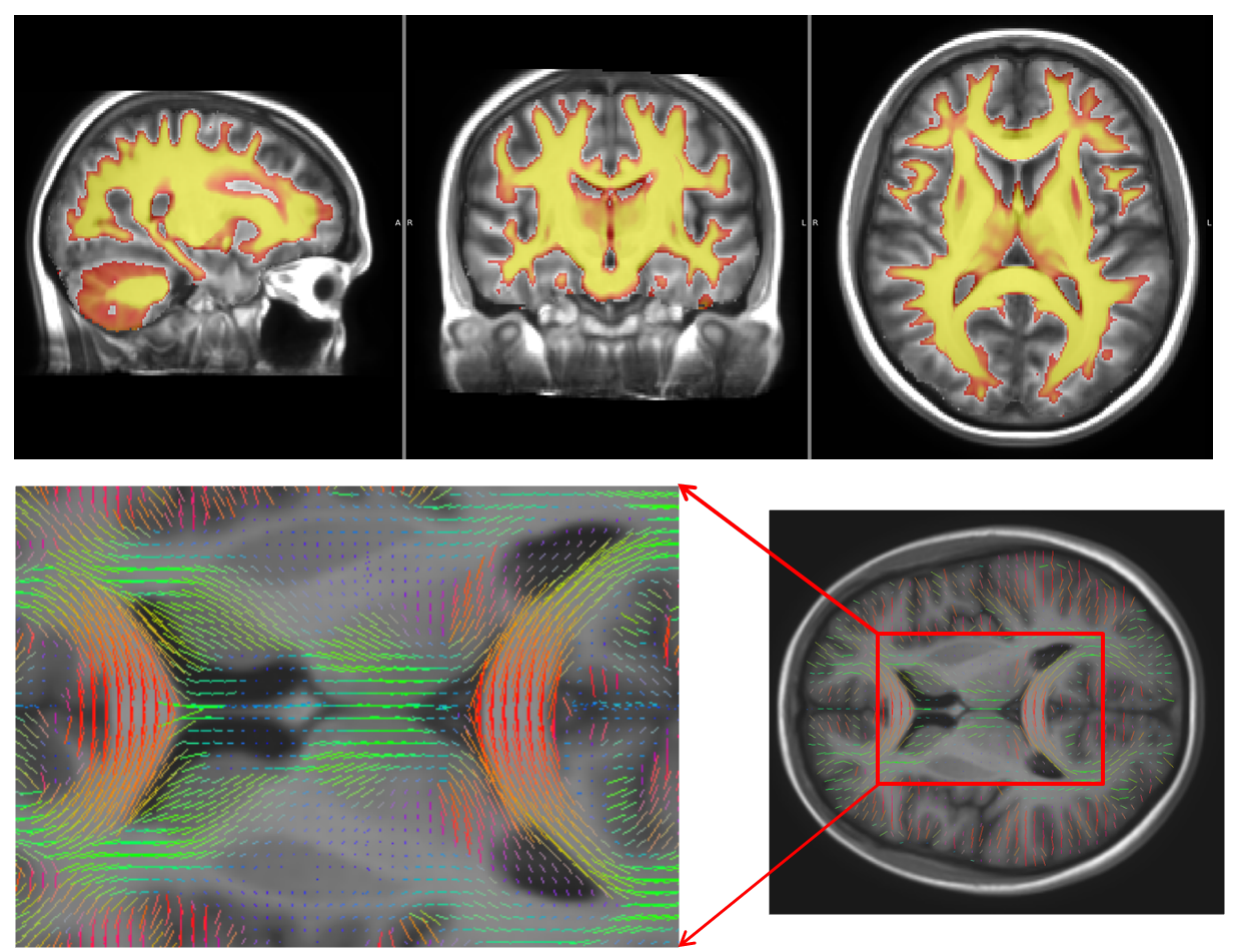

Fig. 3. The multimodal brain template. Top row: FA template overlayed on the T1 template. Bottom row: DTI template is overlayed on top of T1 template. The zoomed section shows good alignment of diffusion tensors along the corpus callosum above and below the ventricles.

are computed from the registered DTIs and the log-Jacobian maps are computed from the deformation field resulting from T1 image registration. Thus, we have a voxelwise multi-channel information. A non-parametric distribution free permutation test is used for comparing the control group against the patients. The test-statistic as suggested in [11] is used for the permutation test.

$$
\begin{aligned}
& T_{n_{1}, n_{2}}=\frac{n_{1} n_{2}}{n_{1}+n_{2}}\left[\frac{1}{n_{1} n_{2}} \sum_{i=1}^{n_{1}} \sum_{j=1}^{n_{2}}\left\|\mathbf{V}_{\mathbf{1 , i}}-\mathbf{V}_{\mathbf{2}, \mathbf{j}}\right\|-\frac{1}{2 n_{1}^{2}} \sum_{i=1}^{n_{1}} \sum_{j=1}^{n_{1}}\left\|\mathbf{V}_{\mathbf{1 , i}}-\mathbf{V}_{\mathbf{1}, \mathbf{j}}\right\|\right. \\
& \left.-\frac{1}{2 n_{2}^{2}} \sum_{i=1}^{n_{2}} \sum_{j=1}^{n_{2}}\left\|\mathbf{V}_{\mathbf{2}, \mathbf{i}}-\mathbf{V}_{\mathbf{2}, \mathbf{j}}\right\|\right]
\end{aligned}
$$

where $\|$.$\| is the Euclidean distance, n_{1}$ and $n_{2}$ are the sizes of control and HAND patients respectively. $\mathbf{V}$ and is the vector of two elements as described above. We used 1000 random permutations to generate the distribution. The figure 5 compares the result of univariate analysis against the multivariate analysis. 


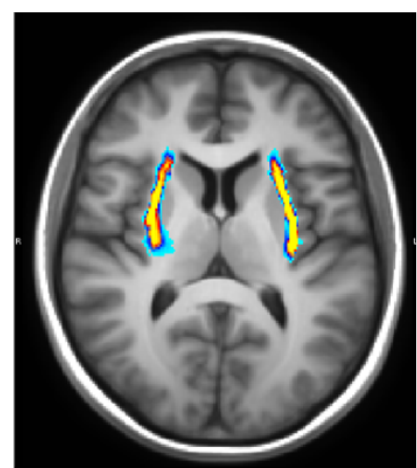

External Capsule

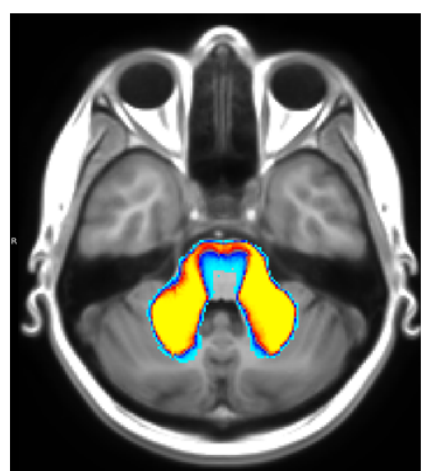

Middle cerebellar peduncle

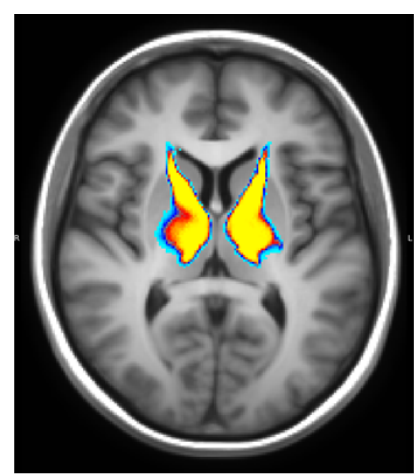

Internal Capsule

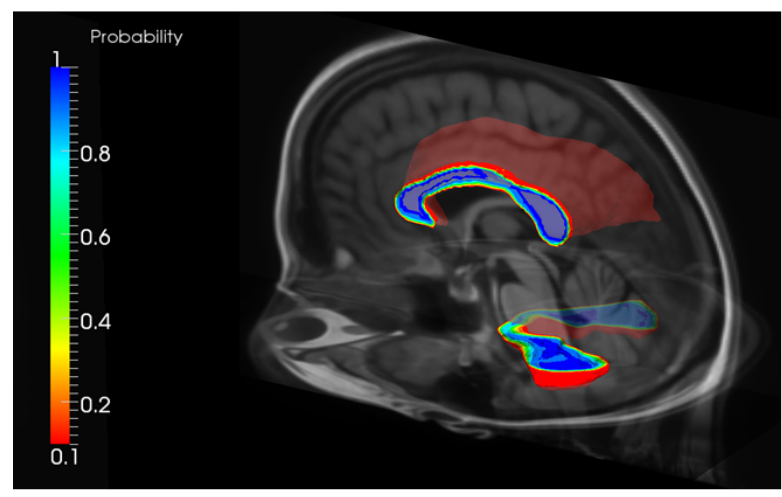

Probabilistic iso-surfaces

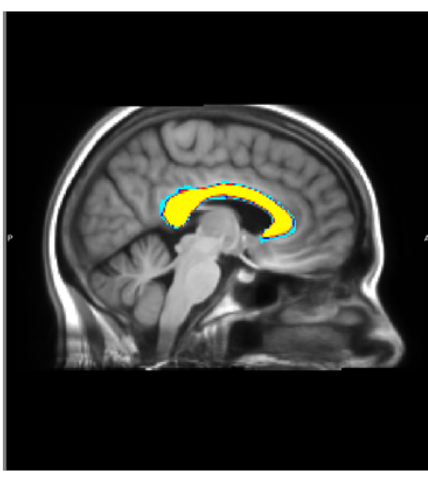

Corpus callosum

Fig. 4. Probabilistic ROIs for some of the representative white matter structures are shown overlayed on the T1 image for anatomical reference. Bottom-right image shows $3 \mathrm{D}$ probabilistic iso-surfaces for corpus callosum and middle cerebral peduncle.

In the top row red-yellow and blue-lightblue shows the statistically significant regions at 5\% significance level for FA and log-Jacobian respectively. The bottom row shows the significantly different regions for multivariate tests. It should be noted that with the multivariate tests, we are able to detect more regions of differences between the controls and HAND patients and thus attributing a higher detection power to such multivariate treatment of images.

\section{Conclusion}

In this paper we have presented a novel workflow for creating a multimodal T1 and DTI template which can be used for population based statistical studies. In our knowledge, there have been very few attempts in the past to perform group study by fusing multiple modalities and making a true multimodal brain atlas. The main idea of the paper is to combine the well accepted existing tools in order 



Univariate Analysis
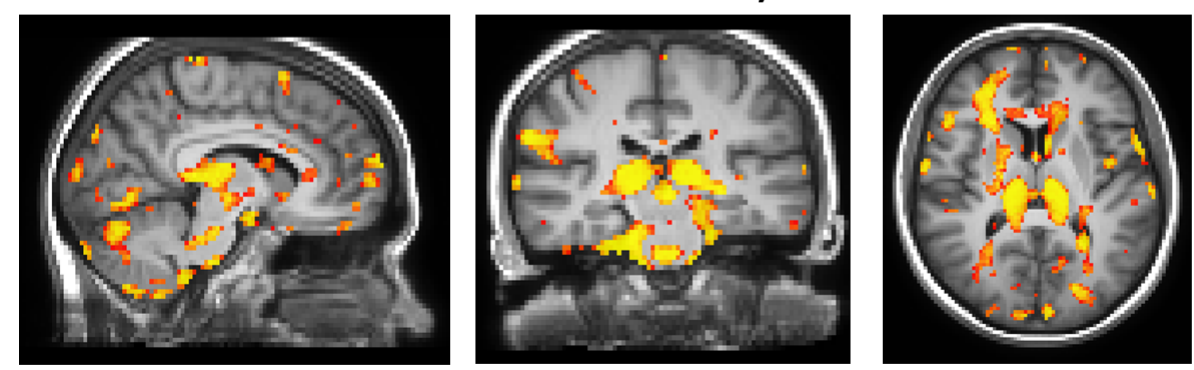

Multivariate Analysis

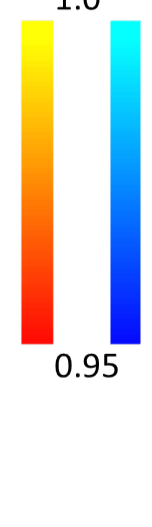

Fig. 5. Statistically significant regions of difference. Top row: Univariate results on FA (red-yellow) and log-Jacobian (blue-lightblue) of the deformation field from T1 registration. Bottom row: Multivariate (red-yellow) statistical test. The images show the test-statistic values between 0.95 and 1 corresponding to $5 \%$ significance level.

to make a workflow and suggest methods for multimodal statistical analysis. We created a probabilistic atlas of white matter regions using the ROI definitions from the WMPM labels. The multimodal atlas will have a wide variety of applications in the future. For example, one can use the probabilistic ROI definition as a prior for manual segmentation. It will be possible to correlate measures across different modalities giving us a better understanding of neuro-pathologies and possibly seek for biomarkers. We already showed an illustration of the possible applications in the case of HAND patients. The multivariate framework seems to have a higher detection power. However, the clinical relevance of these findings remain to be seen. The methods presented in this paper can easily be adopted to create any other population specific multimodal templates. However, we are intending to make this atlas public for research purposes. In the future, it will interesting to look into building a spatio-temporal population specific brain atlases. Such an atlas will give deep insights into disease progression in case of progressive neuro-degenerative diseases like AIDS, Alzheimer's disease and ALS. One of the major contributions of this work is that with the presented method, it is possible to combine well accepted pre-existing tools for creating a population specific multimodal brain atlas and use the same for multimodal statistical 
analysis.

Acknowledgements We would like to acknowledge Nice University Hospital $(\mathrm{CHU})$ and the NEURADAPT study for collecting the data. The work was partly supported by the European Research Council through the ERC Advanced Grant MedYMA 2011-291080.

\section{References}

1. Thompson, P.M., Toga, A.W.: Detection, visualization and animation of abnormal anatomic structure with a deformable probabilistic brain atlas based on random vector field transformations. Medical image analysis 1(4) (1997) 271-294

2. Joshi, S., Davis, B., Jomier, M., Gerig, G.: Unbiased diffeomorphic atlas construction for computational anatomy. NeuroImage 23 (2004) S151-S160

3. Toga, A.W., Thompson, P.M., et al.: Towards multimodal atlases of the human brain. Nature Reviews Neuroscience 7(12) (2006) 952-966

4. Mori, S., et al.: Stereotaxic white matter atlas based on diffusion tensor imaging in an ICBM template. Neuroimage 40(2) (2008) 570-582

5. Guimond, A., Meunier, J., Thirion, J.P.: Average brain models: A convergence study. Computer vision and image understanding 77(2) (2000) 192-210

6. Commowick, O., Malandain, G.: Efficient selection of the most similar image in a database for critical structures segmentation. (2007) 203-210

7. Tustison, N.J., Avants, B.B., et al.: N4ITK: improved N3 bias correction. TMI 29(6) (2010) 1310-1320

8. Lorenzi, M., et al.: LCC-Demons: a robust and accurate symmetric diffeomorphic registration algorithm. NeuroImage 81 (2013) 470-483

9. Fillard, P., Pennec, X., et al.: Clinical DT-MRI estimation, smoothing, and fiber tracking with log-Euclidean metrics. IEEE TMI 26(11) (2007) 1472-1482

10. Arsigny, V., Commowick, O., Pennec, X., Ayache, N.: A log-euclidean framework for statistics on diffeomorphisms. In: MICCAI 2006. Springer (2006) 924-931

11. Baringhaus, L., Franz, C.: On a new multivariate two-sample test. Journal of multivariate analysis 88(1) (2004) 190-206 